\title{
Trace gas sensing using DFB laser and QCL with miniaturized 3D-printed photoacoustic gas cells
}

\author{
Ralf Bauer, Gordon M. H. Flockhart, Walter Johnstone, and Michael Lengden \\ Department of Electronic and Electrical Engineering, University of Strathclyde, Glasgow, UK \\ ralf.bauer@strath.ac.uk
}

\begin{abstract}
Miniaturized 3D-printed photoacoustic trace gas sensors are presented, targeting gas species with absorption features in the telecommunications band as well as the mid-infrared with recovered gas concentrations down to the parts-per-billion range.

OCIS codes: (280.4788) Optical sensing and sensors; (300.6430) Spectroscopy, photothermal
\end{abstract}

\section{Introduction}

Robust, small, cost-effective and sensitive trace gas sensors are a key measurement tool to monitor environmental conditions, industrial process control or biomedical health processes. In all these fields sensitivity levels down to the parts-per-billion (ppb) or even parts-per-trillion (ppt) level are increasingly required to distinguish and quantify gas concentrations of species of interest. The use of photoacoustic spectroscopy (PAS) has shown promise to achieve the required sensitivity levels in small scale packages, with PAS relying on the excitation of an acoustic wave by a modulated light source which is recovered by miniature microphones or other acoustic sensors. Miniature systems have been demonstrated using silicon manufacturing technologies [1], miniature milling [2], or 3D-printing [3, 4]. A further miniaturized approach is the more widely used technique of using qartz tuning forks as acoustic sensors [5].

Here we present our recent work on using 3D-printed miniaturized gas cells combined with commercially available MEMS microphones to achieve trace gas detection of gas species such as $\mathrm{CH}_{4} \mathrm{or}_{2} \mathrm{C}_{2}$. Different light excitation approaches will be presented, using free space mid-infrared laser excitation or fully fibre coupled excitation through DFB lasers, with demonstrated minimum detection capabilities in the ppb range.

\section{Gas sensor design and simulation}

The 3D-printed PAS gas cells (printed on an EnvisionTec Aureus Desktop 3D-printer) consist of a cylindrical resonator with buffer volumes on each side to excite the first acoustic longitudinal resonance of the cell and increase the sensitivity of the sensor (see Fig. 1). The resonator diameter can be tailored to match the width of the excitation laser source; in the near infrared the use of fibre coupled cell designs allows a resonator diameter of $1.8 \mathrm{~mm}$ to match to the free aperture of the graded index (GRIN) fibre collimators, and in the free space cell design, targeted for mid infrared sources, a resonator diameter of $1.5 \mathrm{~mm}$ is typical to match the typical free-space laser beam width. The resonator length for the fibre coupled cell is $10 \mathrm{~mm}$ and for the free space cell $16 \mathrm{~mm}$. The $\lambda / 4$ buffer volumes have a diameter of $10.5 \mathrm{~mm}$ and create the boundary conditions of the acoustic standing wave in the central resonator. An acoustic sensing port is located in the middle of the resonator with a diameter of $0.75 \mathrm{~mm}$ and height of $0.25 \mathrm{~mm}$. This size of this port is matched to the aperture of a MEMS microphone (Wolfson WM7133), that is secured in place using an epoxy resin. The GRIN fibre collimators (Photop KFCS-A-900T-1550-N-100-C-NN) or $0.25 \mathrm{~mm}$ thick calcium fluoride windows are then fixed at the ends of the buffer volumes using the same epoxy to create the optical excitation path and to ensure the cell is fully gas tight.

The acoustic behavior of the gas cells was simulated using COMSOL Multiphysics' thermoacoustics module, to account for thermal and viscous boundary layer losses at the cell walls. A modulated heat source, corresponding to the excitation laser beam, is defined in the center of the cell, with the acoustic port neglected in the first instance to reduce computational requirements of the simulation. An axis-symmetric model is used, with nitrogen as the buffer for the $10 \mathrm{~mm}$ cell, to simulate low concentration (ppb-ppt) measurement, and a buffer gas of methane for the $16 \mathrm{~mm}$ cell to simulate process control applications where high gas concentration measurements are required in a robust, miniature device. The output of this modelling is the determination of the cell resonance frequencies and resonance bandwidth. The resulting temperature distribution in the cell shows the small thermal boundary layers on the resonator walls (see Fig. 2) and its standing wave behavior matches with the pressure distribution in the cell during laser excitation. The simulated resonance frequencies are $15.4 \mathrm{kHz}$ and $12.8 \mathrm{kHz}$ with Q-factors of 51 and 32. It will be shown in the experimental section that the frequencies are a good match to the measured values, while the Q-factors are overestimated by a factor of up to 3.5. This is attributed to the strong dependence of the Q-factor calculations on the values for the bulk viscosity of the gases in the model, with no consistent values available in the literature. The values used here were $2 \cdot 10^{-5} \mathrm{~Pa} \cdot \mathrm{s}$ and $1.4 \cdot 10^{-5} \mathrm{~Pa} \cdot \mathrm{s}$. Additionally, the sidewall roughness of the cells 
is not accounted for and further losses are potentially occurring at the acoustic port and through extra cavities in front of microphone membrane. The general trend and behavior is nevertheless well represented.

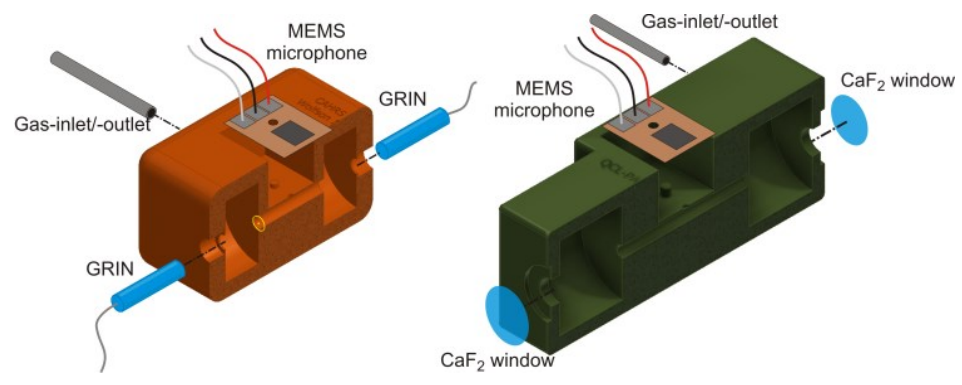

Fig. 1. Design and parts of the fibre-coupled (left) and free space (right) 3D-printed PAS gas cells.
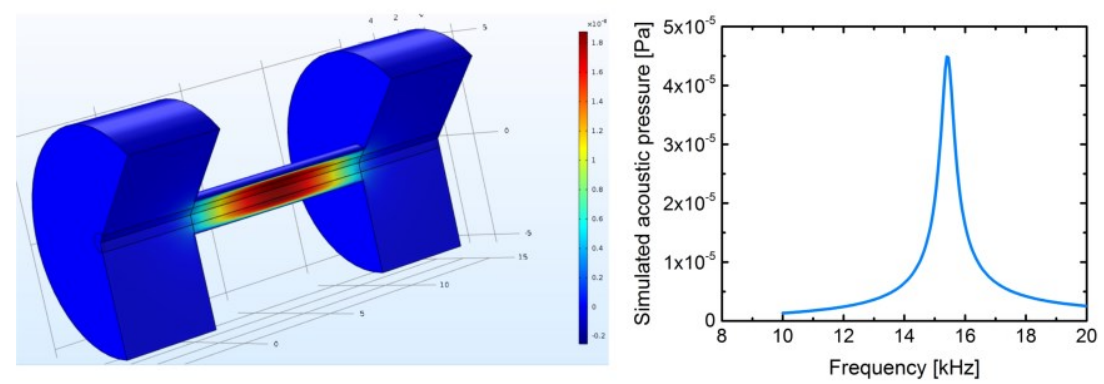

Fig. 2. Simulation of temperature variation distribution at resonance (left) and frequency dependent acoustic pressure (right) of $10 \mathrm{~mm}$ cell.

\section{Experimental results}

A wavelength modulation approach of the excitation laser is used to characterize the achievable detection limits and sensitivities of the two types of gas cells mentioned. For the $10 \mathrm{~mm}$ cell a DFB laser centered at $\sim 1651 \mathrm{~nm}$, in combination with a custom Raman amplifier system delivering a maximum of $1.1 \mathrm{~W}$, is used as the excitation source. The applied modulation consists of a $5 \mathrm{~Hz}$ current ramp to interrogate a spectral feature of interest, a sinusoidal modulation at the cell resonance frequency, and a higher frequency modulation to suppress detrimental nonlinear effects in the fibre amplifier. For the $16 \mathrm{~mm}$ cell a QCL laser, with $4 \mathrm{~mW}$ average output power and center wavelength of $5.26 \mu \mathrm{m}$, is used as the excitation source. The applied modulation again consists of a $5 \mathrm{~Hz}$ ramp and a sinusoidal modulation at the cell resonance frequency, however, in this case there is no necessity for a high frequency modulation to suppress non-linear effects. For both cases the $1^{\text {st }}$ harmonic signal of the microphone is recovered using a SRS 830 lock-in amplifier (LIA) with an integration time of $0.3 \mathrm{~ms}$, combined with a signal capturing using an Agilent DSO5014 oscilloscope. The sample gas used for the $10 \mathrm{~mm}$ cell and Raman amplifier system was $10 \mathrm{ppm} \mathrm{CH}_{4}$ buffered in nitrogen, while the sample gas for the $16 \mathrm{~mm}$ cell and QCL system was $100 \%$ $\mathrm{CH}_{4}$. The resulting recovered gas signals and cell resonance can be seen in Fig. 3. The signal from the Raman amplifier system shows a signal to noise ratio (SNR) of 60, which gives an initial detection limit of $166 \mathrm{ppb}$. With increased integration time this limit can be reduced to $17 \mathrm{ppb}$ at $130 \mathrm{~s}$. The normalized noise equivalent absorption of this cell can be calculated as $4.1 \cdot 10^{-9} \mathrm{~cm}^{-1} \mathrm{~W} \mathrm{~Hz}^{-1 / 2}$. The signal for the QCL shows a SNR of 18 , due to the weak absorption band present for the QCL wavelength. The normalized noise equivalent absorption of this cell is however of the same order of magnitude to the $10 \mathrm{~mm}$ cell with a value of $7.7 \cdot 10^{-9} \mathrm{~cm}^{-1} \mathrm{~W} \mathrm{~Hz}^{-1 / 2}$. The frequency response of the cells provide a resonance frequency of $15.2 \mathrm{kHz}$ for the $10 \mathrm{~mm}$ cell and $12.6 \mathrm{kHz}$ for the $16 \mathrm{~mm}$ cell, with Qfactors of 15 and 12, respectively. The lower Q-factor of the $16 \mathrm{~mm}$ cell has its origin partly in the increased bulk viscosity of methane, compared to the cell content of mostly nitrogen as used for the measurements of the $10 \mathrm{~mm}$ cell. 

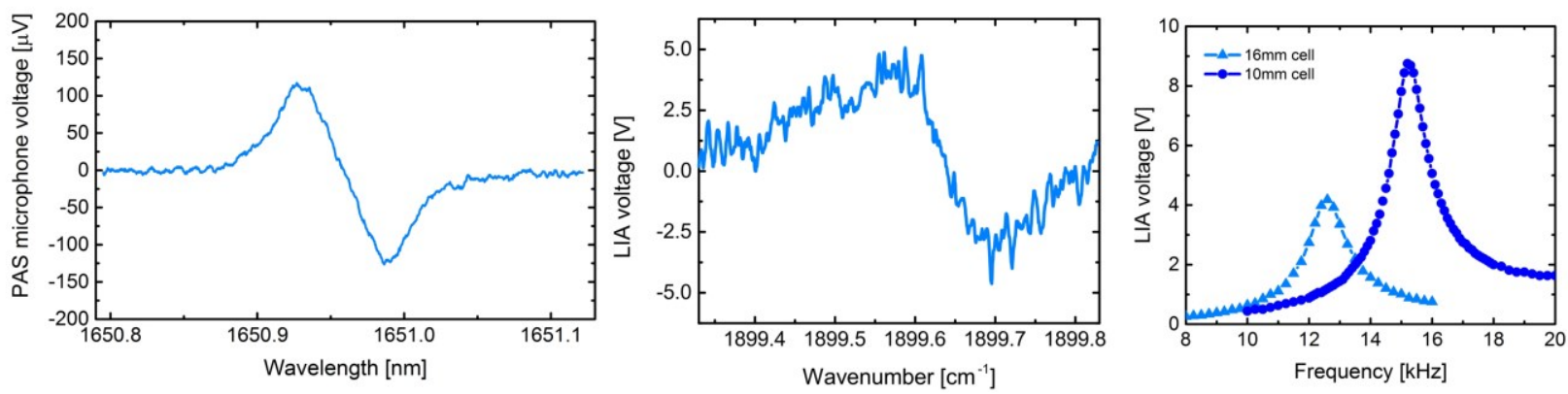

Fig. 3. Measurements of $10 \mathrm{ppm} \mathrm{CH} 4$ at $1651 \mathrm{~nm}$ in $10 \mathrm{~mm}$ cell using the Raman amplifier system (left), measurement of $100 \% \mathrm{CH} 4$ at $5.26 \mu \mathrm{m}$ in $16 \mathrm{~mm}$ cell using the QCL (middle), and cell frequency response for both cases (right).

\section{Conclusions}

This paper has presented our latest work on 3D-printed miniaturized trace gas sensors using PAS. A simulation approach has been described and a comparison to experimental data for optical sources in the near and mid infrared has been shown. We will also present further work on application of these sensors for different gas species as well as additional concepts to increase the functionality of the sensors.

\section{References}

[1] S. L. Firebaugh, K. F. Jensen, and M. A. Schmidt, "Miniaturization and integration of photoacoustic detection," J. Appl. Phys., vol. 92, no. 3, pp. 1555-1563, 2002.

[2] A. L. Ulasevich, A. V. Gorelik, A. A. Kouzmouk, and V. S. Starovoitov, "A compact resonant П-shaped photoacoustic cell with low window background for gas sensing," Appl. Phys. B, vol. 117, no. 2, pp. 549-561, Jun. 2014

[3] R. Bauer, T. Legg, D. Mitchell, G. M. H. Flockhart, G. Stewart, W. Johnstone, and M. Lengden, "Miniaturized Photoacoustic Trace Gas Sensing Using a Raman Fiber Amplifier,” J. Light. Technol., vol. 33, no. 18, pp. 3773-3780, Sep. 2015.

[4] R. Bauer, G. Stewart, W. Johnstone, E. Boyd, and M. Lengden, "3D-printed miniature gas cell for photoacoustic spectroscopy of trace gases," Opt. Lett., vol. 39, no. 16, pp. 4796-4799, Aug. 2014.

[5] P. Patimisco, G. Scamarcio, F. K. Tittel, and V. Spagnolo, "Quartz-enhanced photoacoustic spectroscopy: a review.," Sensors (Basel)., vol. 14, no. 4, pp. 6165-6206, Jan. 2014. 